\title{
RANDOM WALKS AND INDUCED DIRICHLET FORMS ON COMPACT SPACES OF HOMOGENEOUS TYPE
}

\author{
Shi-Lei Kong, Ka-Sing Lau and Ting-Kam Leonard Wong
}

\begin{abstract}
We extend our study of random walks and induced Dirichlet forms on self-similar sets [KLW, KL] to compact spaces of homogeneous type $(K, \rho, \mu)[\mathrm{CW}]$. A successive partition on $K$ brings a natural augmented tree structure $(X, E)$ that is Gromov hyperbolic, and the hyperbolic boundary is Hölder equivalent to $K$. We then introduce a class of transient reversible random walks on $(X, E)$ with return ratio $\lambda$. Using Silverstein's theory of Markov chains, we prove that the random walk induces an energy form on $K$ with

$$
\mathcal{E}_{K}[u] \asymp \iint_{K \times K \backslash \Delta} \frac{|u(\xi)-u(\eta)|^{2}}{V(\xi, \eta) \rho(\xi, \eta)^{\beta}} d \mu(\xi) d \mu(\eta),
$$

where $V(\xi, \eta)$ is the $\mu$-volume of the ball centered at $\xi$ with radius $\rho(\xi, \eta), \Delta$ is the diagonal, and $\beta$ depends on $\lambda$. In particular, for an $\alpha$-set in $\mathbb{R}^{d}$, the kernel of the energy form is of order $\frac{1}{|\xi-\eta|^{\alpha+\beta}}$. We also discuss conditions for this energy form to be a non-local regular Dirichlet form.

\|\|
\end{abstract}

\section{Introduction}

In discrete potential theory [Dy, it is well-known that a transient Markov chain $\left\{Z_{n}\right\}_{n=0}^{\infty}$ on a countable set $X$ converges to a limiting random variable $Z_{\infty}$ on the Martin boundary $\mathcal{M}$, where the union $X \cup \mathcal{M}$ is a compactification of $X$ under a topology involving the Green function. The Martin kernel $K(\cdot, \cdot)$ on $X \times \mathcal{M}$, together with the hitting distribution $\nu$ of $Z_{\infty}$, plays the role of the Poisson kernel in classical analysis: any function $\varphi \in L^{1}(\mathcal{M}, \nu)$ can be extended to a harmonic function on the "interior" $X$ via

$$
(H \varphi)(x)=\int_{\mathcal{M}} \varphi(\xi) K(x, \xi) d \nu(\xi), \quad x \in X .
$$

Here harmonicity is defined in terms of the transition probability of the chain.

2010 Mathematics Subject Classification. Primary 60J10; Secondary 28A80, 60J50.

Keywords: Dirichlet form, compact space of homogeneous type, hyperbolic graph, Martin boundary,

Naïm kernel, reversible random walk.

The research is supported in part by the HKRGC grant and SFB 1283 of the German Research Council. 
The Markov chains we consider in this paper are always reversible: with a discrete energy form $\mathcal{E}_{X}$ on $X$ given by

$$
\mathcal{E}_{X}[u]=\frac{1}{2} \sum_{x, y \in X} c(x, y)|u(x)-u(y)|^{2},
$$

where $c(x, y)=c(y, x) \geq 0$ and $m(x):=\sum_{y \in X} c(x, y)<\infty$, the corresponding reversible Markov chain on $X$ is the one with transition probability $P(x, y)=c(x, y) / m(x)$. In [Si], Silverstein showed that for such a Markov chain, the induced energy form $\mathcal{E}_{\mathcal{M}}$ on $\mathcal{M}$, defined by

$$
\mathcal{E}_{\mathcal{M}}[\varphi]=\mathcal{E}_{X}[H \varphi]
$$

has the expression

$$
\mathcal{E}_{\mathcal{M}}[\varphi]=C \iint_{\mathcal{M} \times \mathcal{M} \backslash \Delta}|\varphi(\xi)-\varphi(\eta)|^{2} \Theta(\xi, \eta) d \nu(\xi) d \nu(\eta),
$$

where $C>0$ is a constant and $\Delta$ denotes the diagonal. Here $\Theta(\cdot, \cdot)$ is called the Naïm kernel [Na. This has an analog in classical analysis [FOT]: for the Brownian motion on the closed unit disk $\mathbb{D}$ started at the center and reflected on the boundary $\mathbb{T}=\partial \mathbb{D}$, there is an induced jump process (Cauchy process) on $\mathbb{T}$ such that the corresponding energy form on $\mathbb{T}$ has the expression in (1.2) with $\Theta(\xi, \eta)=\sin ^{-2}\left(\frac{\xi-\eta}{2}\right) \asymp|\xi-\eta|^{-2}$. (For $f, g>0$, by $f \asymp g$, we mean $C^{-1} g \leq f \leq C g$ for some $C>0$.)

The above theoretical setup leads to many challenging questions (e.g., see [CF, Ki2, BGPW]). Among them we are interested in the following two problems:

(i) Study those Markov chains that the Martin boundaries and the hitting distributions can be identified with some familiar sets and measures respectively.

(ii) With the reversible Markov chains satisfying (i), find explicit expressions or estimates of the Naïm kernels in (1.2), and to study the associated Dirichlet forms.

These problems are difficult in general as it is non-trivial to estimate the Green function and the hitting probabilities. For random walks on discrete groups, the identification problem in (i) has been studied for a long time, and the reader can refer to Ka1 for an informative survey. More recent attempts deal with self-similar sets in fractal geometry. For such a set $K$ generated by an iterated function system (IFS), there is a symbolic space (coding space) $\Sigma^{*}$ which gives a convenient symbolic representation of any $\xi \in K$. If the IFS satisfies the open set condition (OSC), then the representation is unique for generic points of $K$. There are a number of studies of random walks on $\Sigma^{*}$ such that $K$ can be identified with the Martin boundary $\mathcal{M}$ [DoSn, JuLW], Ka2, LN1, LN2, LW2]. The most far reaching attempt is due to Kaimanovich [Ka2]: he introduced a natural augmented tree for the Sierpiński gasket $K$ by adding horizontal edges on $\Sigma^{*}$ according to the intersections of the cells at each level (Sierpiński graph). He showed that the Sierpiński graph is hyperbolic in the sense of Gromov $\mathrm{Gr}$ and the hyperbolic boundary is Hölder 
equivalent to $K$. In [LW1, LW2, construction of the augmented tree and identification of the boundary were extended to all self-similar sets. These works brought into play the hyperbolic structure and hyperbolic boundary which are very useful tools for studying analysis on fractals through random walks.

In this respect, the current authors introduced in [KLW] a class of reversible random walks $\left\{Z_{n}\right\}_{n=0}^{\infty}$ that have constant return ratio $0<\lambda<1$ on the augmented tree of a self-similar set $K$ with the OSC. We call this walk a $\lambda$-natural random walk ( $\lambda$-NRW, see Section 3). By using a theorem of Ancona [An], it was shown that the Martin boundary of $\left\{Z_{n}\right\}_{n=0}^{\infty}$ can be identified with the hyperbolic boundary, and thus the self-similar set $K$ as well. Moreover, by estimating the ever-visiting probability of $\left\{Z_{n}\right\}_{n=0}^{\infty}$ on states in $X$ and on $\mathcal{M}$ in terms of the Gromov product in the hyperbolic graph, we obtained explicit expression of the hitting distribution as well as estimates of the Martin kernel and the Naïm kernel. In particular, the Naïm kernel satisfies $\Theta(\xi, \eta) \asymp|\xi-\eta|^{-(\alpha+\beta)}$, where $\alpha$ is the Hausdorff dimension of $K$, and $\beta=\frac{\log \lambda}{\log r}$ and $r$ is the minimal contraction ratio of the IFS.

The main purpose of this paper is to extend the considerations in [KLW,, $\mathrm{KL}$ ] to the class of compact spaces of homogeneous type, and summarize some of the results. A space of homogeneous type is a triple $(M, \rho, \mu)$ where $M$ is a set, $\rho$ is a quasi-metric and $\mu$ is a regular Borel measure with the doubling property. This class of spaces was introduced by Coifman and Weiss in [CW], and has been studied in great detail in the theories of metric measure spaces as well as $H^{p}$ spaces, singular integrals and analytic capacities (see $[\mathrm{AM}, \mathrm{DH}, \mathrm{LuS}]$ and the references therein). This class contains the classical domains, the $\alpha$-sets [JoWa, and many more examples (see [CW]). In [Ch], Christ showed that a space of homogeneous type admits a partition system (dyadic cubes) which can be represented by a tree. When $M=K$ is compact, similar to the symbolic space of the selfsimilar sets, we can augment this tree by adding suitable horizontal edges corresponding to neighboring cells, and the resulting graph (augmented tree) is hyperbolic. We can extend the above $\lambda$-NRW to this situation, and obtain similar identification results and estimates of the kernels. The induced energy form in this case has the estimate

$$
\mathcal{E}_{K}^{(\beta)}[u] \asymp \iint_{K \times K \backslash \Delta} \frac{|u(\xi)-u(\eta)|^{2}}{V(\xi, \eta) \rho(\xi, \eta)^{\beta}} d \mu(\xi) d \mu(\eta),
$$

where $V(\xi, \eta)$ is the $\mu$-volume of the ball center at $\xi$ with radius $\rho(\xi, \eta)$, and $\beta$ depends on $\lambda$.

The domain of the induced energy form $\mathcal{E}_{K}^{(\beta)}$ is a Besov space $\Lambda_{2,2}^{\beta / 2}$. By the polarization identity, $\mathcal{E}_{K}^{(\beta)}$ defines a non-local regular Dirichlet form if $\Lambda_{2,2}^{\beta / 2} \cap C(K)$ is dense in $C(K)$ and in $\Lambda_{2,2}^{\beta / 2}$. The family $\left\{\Lambda_{2,2}^{\beta / 2}\right\}_{\beta>0}$ is decreasing in $\beta$, and in general it is trivial for large $\beta$. This defines a critical exponent $\beta^{*}$. It is an important index because in the standard cases, it is the place where a local Dirichlet form arises (which corresponds to a Laplacian) with domain $\Lambda_{2, \infty}^{\beta^{*} / 2}$ (while $\Lambda_{2,2}^{\beta^{*} / 2}$ contains only constant functions). For example for classical 
domains we have $\beta^{*}=2$, and for the Sierpiński gasket $\beta^{*}=\log 5 / \log 2$. We will give some discussion on this, and provide a criterion to determine the critical exponents on the p.c.f. self-similar sets as in $[\mathrm{KL}$.

The proofs of the results will appear separately; for the special case on the self-similar sets, the reader can refer to $[\mathrm{LW}, \mathrm{KLW}, \mathrm{KL}$. The rest of the paper is organized as follows. We first recall, in Section 2, some preliminaries about hyperbolic graphs and the boundary theory of Markov chains. In Section 3, we study the partition systems and the tree structures for compact spaces of homogeneous type, and establish the hyperbolicity of the augmented trees. In Section 4, we investigate the $\lambda$-NRW on the augmented trees; we identify the underlying set with the hyperbolic boundary and the Martin boundary. The estimations of the Martin kernel and the Naïm kernel are stated. Finally, in Section 5 we discuss the induced Dirichlet forms and a criterion of the critical exponents.

\section{Preliminaries}

Let $(X, E)$ be a countably infinite connected graph with an edge set $E$ and a root $\vartheta \in X$. For $x \in X$, we let $|x|:=d(\vartheta, x)$ where $d(\cdot, \cdot)$ is the graph distance, and let $\mathcal{J}_{n}=\{x \in X$ : $|x|=n\}$ be the $n$-th level of the graph. We define a partial order $\prec$ on $X$ with $y \prec x$ if $x$ lies on some geodesic path $\pi(\vartheta, y)$; for $m \geq 0$, and define the $m$-th descendant set of $x$ as $\mathcal{J}_{m}(x):=\{y \in X: y \prec x$ and $|y|=|x|+m\}$.

Let $E_{v}:=\{(x, y) \in E:|x|-|y|= \pm 1\}$ and $E_{h}:=\{(x, y) \in E:|x|=|y|\}$ denote the vertical edge set and the horizontal edge set respectively. Clearly $E=E_{v} \cup E_{h}$. We call $(X, E)$ a (rooted) tree if $E=E_{v}$ and any vertex $x \in X \backslash\{\vartheta\}$ has a unique parent $x^{-}$that satisfies $x \in \mathcal{J}_{1}\left(x^{-}\right)$.

To discuss the hyperbolicity of an infinite rooted graph $(X, E)$, let us recall the setup by Gromov $\mathrm{Gr}$ (see also $[\mathrm{Wo}$ ). The Gromov product of two vertices $x, y \in X$ is $(x \mid y):=$ $\frac{1}{2}(|x|+|y|-d(x, y))$. If there exists $\delta \geq 0$ such that $(x \mid y) \geq \min \{(x \mid z),(z \mid y)\}-\delta$ for all $x, y, z \in X$, then we say that $(X, E)$ is (Gromov) hyperbolic. Clearly every tree is hyperbolic with $\delta=0$. For $a>0$, we define the Gromov metric $\varrho_{a}(\cdot, \cdot)$ on $X$ by

$$
\varrho_{a}(x, y)= \begin{cases}e^{-a(x \mid y)}, & \text { if } x \neq y \\ 0, & \text { if } x=y\end{cases}
$$

Then $\varrho_{a}(x, y) \leq e^{\delta a} \max \left\{\varrho_{a}(x, z), \varrho_{a}(z, y)\right\}$ (hence $\varrho_{a}$ is a quasi-metric as defined in (3.1) ); this $\varrho_{a}$ is equivalent to a metric when $e^{\delta a}<\sqrt{2}$.

Definition 2.1. For $a>0$, let $\widehat{X}_{H}$ denote the $\varrho_{a}$-completion of $X$, and call $\partial_{H} X:=$ $\widehat{X}_{H} \backslash X$ the hyperbolic boundary of $(X, E)$. 
We note that the topology does not depend on the value of $a$. We treat $\xi \in \partial_{H} X$ as the limit of a $\rho_{a}$-Cauchy sequence $\left\{x_{n}\right\}_{n=0}^{\infty}$. In particular, we can take $\left\{x_{n}\right\}_{n=0}^{\infty}$ to be a geodesic ray $\left[x_{0}, x_{1}, \cdots\right]$ (i.e., each finite segment is a geodesic of the ambient graph $(X, E))$. We denote such a ray by $\pi\left(x_{0}, \xi\right)$. We extend the partial order $\prec$ by writing $\xi \prec x$ if $x$ lies on some $\pi(\vartheta, \xi)$, and it is easy to define geodesics for $x \in X, \xi \in \partial_{H} X$, and (bi-infinite) geodesics for $\xi, \eta \in \partial_{H} X$.

For the augmented tree discussed in the next section, we can extend the Gromov product to $(\xi \mid \eta), \xi, \eta \in \partial_{H} X$ (see (3.3)), then we can use this to define $\varrho_{a}(\cdot, \cdot)$ on $\widehat{X}_{H}$ in the same way as in (2.1), and it is still a quasi-metric.

In [Ka2, Kaimanovich introduced a class of rooted graphs $(X, E)$ that we call preaugmented trees: the subgraph $\left(X, E_{v}\right)$ is a tree, and

$$
(x, y) \in E_{h} \Rightarrow x^{-}=y^{-} \text {or }\left(x^{-}, y^{-}\right) \in E_{h} .
$$

The pre-augmented tree allows us to have a simple expression of its geodesics. For two distinct $x, y$ in $X$, there exists a geodesic consisting of three segments: $\pi(x, u), \pi(u, v)$ and $\pi(v, y)$, where $u, v$ satisfy $x \prec u, y \prec v,|u|=|v|$ (hence the middle one is horizontal, and the other two are vertical). In this paper we always use geodesics of this form unless otherwise stated. Note that for $x, y \in X$, the geodesic $\pi(x, y)$ is not necessary unique, and we call the one whose horizontal part satisfies $\pi(u, v) \subset \mathcal{J}_{n}$ with $n$ smallest a canonical geodesic between $x$ and $y$.

The following is a useful criterion for a pre-augmented tree to be hyperbolic.

Proposition 2.2. [LW1, Theorem 2.3] A pre-augmented tree is hyperbolic if and only if there exists $M<\infty$ such that the lengths of all horizontal geodesic segments are bounded by $M$.

For a hyperbolic pre-augmented tree, it is direct to show that for $\xi, \eta \in \partial_{H} X$, there is a canonical geodesic $\pi(\xi, \eta)$.

Next we consider random walks and their Martin boundaries. An (electric) network $(X, E, c)$ is a locally finite connected graph $(X, E)$ together with a nonnegative function $c$ on $X \times X$ that satisfies $c(x, y)=c(y, x)>0$ if and only if $(x, y) \in E$; we call such $c(x, y)$ the conductance of the edge $(x, y)$, and $m(x):=\sum_{y \in X} c(x, y)>0$ the total conductance at $x$. Let $\ell(X)$ denote the collection of real functions on $X$. The graph energy of $f \in \ell(X)$ is defined by

$$
\mathcal{E}_{X}[f]=\frac{1}{2} \sum_{x, y \in X} c(x, y)|f(x)-f(y)|^{2},
$$

and the domain of $\mathcal{E}_{X}$ is $\mathcal{D}_{X}=\left\{f \in \ell(X): \mathcal{E}_{X}[f]<\infty\right\}$. For $X^{\prime} \subset X$, a function $f \in \ell(X)$ is said to be harmonic on $X^{\prime}$ if $\sum_{y \in X} c(x, y)(f(x)-f(y))=0$ for any $x \in X^{\prime}$. We define the effective resistance between two disjoint finite nonempty subsets $F, G \subset X$ by

$$
R_{X}(F, G)=\left(\min \left\{\mathcal{E}_{X}[f]: f \in \ell(X) \text { with } f=1 \text { on } F \text {, and } f=0 \text { on } G\right\}\right)^{-1} .
$$


Also set $R_{X}(F, G)=0$ if $F \cap G \neq \emptyset$ by convention. Clearly the energy minimizer in (2.3) is unique, and is harmonic on $X \backslash(F \cup G)$.

Every conductance $c$ defines a reversible random walk $\left\{Z_{n}\right\}_{n=0}^{\infty}$ on the graph $(X, E)$, in which the transition probability is given by $P(x, y)=\frac{c(x, y)}{m(x)}, x, y \in X$. In this study we always fix $\vartheta \in X$ so that $(X, E)$ is a rooted graph, and assume that $\left\{Z_{n}\right\}$ is transient, i.e., its Green function $G(x, y):=\sum_{n=0}^{\infty} P^{n}(x, y)<\infty$ for all $x, y \in X$. Write $\mathbb{P}\left(\cdot \mid Z_{0}=\right.$ $x)=\mathbb{P}_{x}(\cdot)$ for short. The ever-visiting probability is defined as $F(x, y):=\mathbb{P}_{x}\{\exists n \geq$ 0 such that $Z_{n}=y$; ; it satisfies $G(x, y)=F(x, y) G(y, y)$. We define the Martin kernel by

$$
K(x, y)=\frac{G(x, y)}{G(\vartheta, y)}=\frac{F(x, y)}{F(\vartheta, y)}, \quad x, y \in X .
$$

Definition 2.3. We call $\mathcal{M}=\widehat{X} \backslash X$ the Martin boundary of $\left\{Z_{n}\right\}$, where $\widehat{X}$ is the compactification of $X$ such that $K(x, \cdot)$ extends continuously to $\widehat{X}$ for all $x \in X$.

Under the Martin topology, the random walk $\left\{Z_{n}\right\}$ converges to an $\mathcal{M}$-valued random variable $Z_{\infty}$ almost surely. The hitting distribution $\nu$ is defined by $\nu(B)=\mathbb{P}_{\vartheta}\left(Z_{\infty} \in B\right)$ for any Borel set $B \subset \mathcal{M}$. For $\xi \in \mathcal{M}$, define the $\xi$-process to be the random walk $\left\{Z_{n}^{\xi}\right\}_{n=0}^{\infty}$ on $(X, E)$ with the transition probability $P^{\xi}(x, y):=P(x, y) K(y, \xi) / K(x, \xi)$; here $P^{\xi}$ is stochastic since $K(\cdot, \xi)$ is $P$-harmonic. Analogously we define the hitting distribution $\nu^{\xi}$ for the $\xi$-process. The minimal Martin boundary of $\left\{Z_{n}\right\}$ is given by $\mathcal{M}_{\text {min }}:=\left\{\xi \in \mathcal{M}: \nu^{\xi}\right.$ is the point mass at $\left.\xi\right\}$, and satisfies $\nu\left(\mathcal{M} \backslash \mathcal{M}_{\min }\right)=0$.

For $F \subset X$, let $m(F):=\sum_{x \in F} m(x)$ and let $c(\partial F):=\sum_{x \in F, y \notin F} c(x, y)$. We say that a conductance $c$ on $(X, E)$ has strong isoperimetry (SI) if

$$
\sup \left\{\frac{m(F)}{c(\partial F)}: F \subset X \text { and } F \text { is finite }\right\}<\infty .
$$

Note that (SI) implies the transience of $\left\{Z_{n}\right\}$, but the converse is not true. As an alternative version of Ancona's Theorem in $[\mathrm{An}$, we have

Theorem 2.4. (Ancona) Let $(X, E)$ be a hyperbolic graph. Suppose a conductance c on $(X, E)$ satisfies hypotheses (SI) and $\inf _{(x, y) \in E} P(x, y)>0$. Then the reversible random walk $\left\{Z_{n}\right\}_{n=0}^{\infty}$ satisfies

$$
F(x, y) \asymp F(x, z) F(z, y)
$$

uniformly for all $x, y, z \in X$ such that $z$ lies on some $\pi(x, y)$. Moreover, the Martin boundary $\mathcal{M}$ equals the minimal Martin boundary $\mathcal{M}_{\min }$, and is homeomorphic to the hyperbolic boundary $\partial_{H} X$.

We define the Poisson integral $H: L^{1}(\mathcal{M}, \nu) \rightarrow \ell(X)$ by

$$
(H u)(x)=\int_{\mathcal{M}} K(x, \xi) u(\xi) d \nu(\xi), \quad u \in L^{1}(\mathcal{M}, \nu), x \in X
$$


Since $K(\cdot, \xi)$ is harmonic for all $\xi \in \mathcal{M}$, we know that $H u$ is harmonic on $X$. Via the operator $H$, the energy form $\left(\mathcal{E}_{X}, \mathcal{D}_{X}\right)$ in (2.2) induces a bilinear form $\left(\mathcal{E}_{\mathcal{M}}, \mathcal{D}_{\mathcal{M}}\right)$ on $L^{2}(\mathcal{M}, \nu)$ defined by

$$
\mathcal{E}_{\mathcal{M}}(u, v)=\mathcal{E}_{X}(H u, H v), \quad u, v \in \mathcal{D}_{\mathcal{M}},
$$

where the domain $\mathcal{D}_{\mathcal{M}}=\left\{u \in L^{2}(\mathcal{M}, \nu): H u \in \mathcal{D}_{X}\right\}$. Define the Nä̈m kernel by

$$
\Theta(x, y)=\frac{K(x, y)}{G(x, \vartheta)}=\frac{F(x, y)}{F(x, \vartheta) G(\vartheta, \vartheta) F(\vartheta, y)}, \quad x, y \in X .
$$

This kernel is symmetric on $X \times X$, and can be extended continuously to $\Theta(x, \eta)$ on $X \times \mathcal{M}$ as the Martin kernel $K(x, y)$ does. The extension to $\Theta(\xi, \eta)$ for $\xi, \eta \in \mathcal{M}$ is more involved. With the notations in the rooted graph $(X, E)$, for $m \geq 0$ and $z \in X$, let $\tau_{m}^{*}$ be the last visit time of $\mathcal{J}_{m}$ by the $\xi$-process $\left\{Z_{n}^{\xi}\right\}$, i.e., $\tau_{m}^{*}=\sup \left\{n \geq 0:\left|Z_{n}^{\xi}\right|=m\right\}$, and let $\ell_{m}^{\xi}(z)=\mathbb{P}_{\vartheta}^{\xi}\left(Z_{\tau_{m}^{*}}^{\xi}=z\right)$. The following extension of $\Theta(\cdot, \cdot)$ on $\mathcal{M} \times \mathcal{M} \backslash \Delta$ (here $\Delta:=\{(\xi, \xi): \xi \in \mathcal{M}\})$ was introduced by Silverstein in [Si]:

$$
\Theta(\xi, \eta)=\lim _{m \rightarrow \infty} \sum_{z \in \mathcal{J}_{m}}{ }_{m}^{\xi}(z) \Theta(z, \eta), \quad \xi \neq \eta \in \mathcal{M}
$$

This limit exists since the sum is increasing in $m$. One of the main results in [Si] is stated below.

Theorem 2.5. (Silverstein) The induced form $\left(\mathcal{E}_{\mathcal{M}}, \mathcal{D}_{\mathcal{M}}\right)$ in (2.6) has the expression

$$
\begin{aligned}
& \mathcal{E}_{\mathcal{M}}(u, v)=\frac{m(\vartheta)}{2} \iint_{\mathcal{M} \times \mathcal{M} \backslash \Delta}(u(\xi)-u(\eta))(v(\xi)-v(\eta)) \Theta(\xi, \eta) d \nu(\xi) d \nu(\eta), \quad u, v \in \mathcal{D}_{\mathcal{M}} . \\
& \text { Moreover, } \mathcal{D}_{\mathcal{M}}=\left\{u \in L^{2}(\mathcal{M}, \nu): \mathcal{E}_{\mathcal{M}}[u]:=\mathcal{E}_{\mathcal{M}}(u, u)<\infty\right\} .
\end{aligned}
$$

\section{Partition and augmented tree}

The aim of this section is to associate every compact space of homogeneous type $(K, \rho, \mu)$ with some hyperbolic graph $(X, E)$ such that the hyperbolic boundary $\left(\partial_{H} X, \varrho_{a}\right) \approx(K, \rho)$. Recall that a quasi-metric $\rho$ on a set $M$ is a function on $M \times M$ to $[0, \infty)$ that satisfies the conditions of a metric except that the triangle inequality is weakened [AM]: for some $C_{\rho} \geq 1$

$$
\rho(\xi, \eta) \leq C_{\rho}(\rho(\xi, \zeta)+\rho(\zeta, \eta)), \quad \forall \xi, \eta, \zeta \in M
$$

A quasi-metric $\rho$ defines a topology $\mathcal{T}_{\rho}$ on $M$ by

$$
\mathcal{T}_{\rho}=\{\Omega \subset M: \forall \xi \in \Omega, \exists \varepsilon>0 \text { s.t. } B(\xi, \varepsilon):=\{\eta \in M: \rho(\xi, \eta)<\varepsilon\} \subset \Omega\} .
$$

Note that for some $\rho$, not all balls $B(\xi, r)$ are open with respect to $\mathcal{T}_{\rho}$. However, Macias and Segovia [MS] proved that there exists a quasi-metric $\rho^{\prime}$, equivalent to $\rho$ in the sense that $\rho^{\prime}(\xi, \eta) \asymp \rho(\xi, \eta)$ (hence $\mathcal{T}_{\rho^{\prime}}=\mathcal{T}_{\rho}$ ), such that all associated metric balls belong to $\mathcal{T}_{\rho}$. Without loss of generality we will assume that metric balls are open throughout the paper. 
Definition 3.1. A space of homogeneous type is a set $M$ equipped with a quasi-metric $\rho$ for which all balls $B(\xi, r)$ are open, and a non-negative regular Borel measure $\mu$ that satisfies the doubling condition: there exists $C>0$ such that

$$
0<\mu(B(x, 2 r)) \leq C \mu(B(x, r))<\infty, \quad \forall \xi \in M, r>0 .
$$

Typical examples of spaces of homogeneous type include classical Euclidean domains, Riemannian manifolds and homogeneous spaces. A non-isotropic example is $M=\mathbb{R}^{d}$ with the Lebesgue measure $\mu$ and $\rho(x, y)=\sum_{i=1}^{d}\left|x_{i}-y_{i}\right|^{s_{i}}$, where $s_{1}, \cdots, s_{d}$ are positive numbers. For more examples and a general method for constructing these spaces, the reader can refer to $\mathrm{CW}$. In fractal geometry, an important class of examples is given by the $\alpha$-sets, which are closed subsets in $\mathbb{R}^{d}$ equipped with the Euclidean distance and the Ahlfors $\alpha$-regular measures $\mu$, i.e., $\mu(B(x, r)) \asymp r^{\alpha}$ for all $x \in K$ and $r \in(0,1)$.

Through out this paper we will use $(K, \rho, \mu)$ to denote a compact space of homogeneous type unless otherwise specified. For convenience, we assume that $\operatorname{diam}(K):=$ $\sup _{x, y \in K} \rho(x, y)=1$ and $\mu$ is a probability measure. We first introduce a tree of partitions on the space as in [LW2].

Definition 3.2. Let $\mathcal{K}$ denote the collection of nonempty compact subsets of $K$. We call a triple $\left(X, E_{v}, \Phi\right)$ an index tree of $(K, \rho, \mu)$ if $\left(X, E_{v}\right)$ is a tree together with a set-valued mapping $\Phi: X \rightarrow \mathcal{K}$ that satisfies the following:

(i) $\Phi(\vartheta)=K$, and $\Phi(x)=\bigcup_{y \in \mathcal{J}_{1}(x)} \Phi(y)$ for all $x \in X$;

(ii) $\exists b, c>0$ and $r_{0} \in(0,1)$ such that each $x \in X$ satisfies $B\left(\xi_{x}, b r_{0}^{|x|}\right) \subset \Phi(x) \subset$ $B\left(\xi_{x}, c r_{0}^{|x|}\right)$ for some $\xi_{x} \in K$;

(iii) ( $\mu$-separation) for distinct $x, y \in X$ with $|x|=|y|, \mu(\Phi(x) \cap \Phi(y))=0$.

The parameter $r_{0}$ in (ii) is referred to as the contraction ratio of $\Phi$. Note that for each $\xi \in K$, there exists a sequence of $x_{n} \in \mathcal{J}_{n}$ such that $\xi \in \Phi\left(x_{n}\right)$ for all $n$, which yields $\{\xi\} \in \bigcap_{n=0}^{\infty} \Phi\left(x_{n}\right)$, and we call $\left\{x_{n}\right\}_{n=0}^{\infty}$ a representation of $\xi \in K$.

We first show that a compact space of homogeneous type always admits an index tree according to the above definition. This follows from the construction of dyadic cubes by Christ $\mathrm{Ch}$.

Example 3.3. Dyadic cubes For $\varepsilon>0$, we define an $\varepsilon$-net on $K$ as a finite subset $\Xi \subset K$ such that $\bigcup_{\xi \in \Xi} B(\xi, \varepsilon)=K$, and $\rho(\xi, \eta) \geq \varepsilon$ if $\xi, \eta \in \Xi$ with $\xi \neq \eta$. Assume that $K$ has diameter 1 , and fix $r_{0} \in(0,1)$ and $b>0$ such that $\frac{C_{\rho}^{3} r_{0}}{1-C_{\rho} r_{0}}+C_{\rho}^{2} b \leq \frac{1}{2}$. For $m \geq 0$, let $\Xi_{m}$ be an $r_{0}^{m}$-net on $K$, and index its elements by $\mathcal{J}_{m}$ so chosen that the $\mathcal{J}_{m}$ 's are disjoint; then we write $\Xi_{m}=\left\{\xi_{x}\right\}_{x \in \mathcal{J}_{m}}$.

For $m \geq 1$ and $x \in \mathcal{J}_{m}$, we choose $x^{-} \in \mathcal{J}_{m-1}$ such that $\xi_{x^{-}}$is the nearest point from $\xi_{x}$ among all points in $\Xi_{m-1}$ (if there are two or more nearest points, we select an arbitrary one from them); clearly $\rho\left(\xi_{x}, \xi_{x^{-}}\right) \leq r_{0}^{m-1}$. Let $X=\bigcup_{m=0}^{\infty} \mathcal{J}_{m}$ and let 
$E_{v}=\left\{\left(x, x^{-}\right),\left(x^{-}, x\right): x \in X, x \neq \vartheta\right\}$. Then $\left(X, E_{v}\right)$ is a tree that satisfies $\mathcal{J}_{1}(x) \neq \emptyset$ for every $x \in X$. We define

$$
\Phi^{\circ}(x)=\bigcup_{z: z \prec x} B\left(\xi_{z}, b r_{0}^{|z|}\right) \quad \text { and } \quad \Phi(x)=\overline{\Phi^{\circ}(x)}, \quad x \in X,
$$

and call $\{\Phi(x)\}_{x \in X}$ a set of dyadic cubes, where $\prec$ is the partial order defined in the last section. Then it is clear that $K=\overline{\Phi^{\circ}(\vartheta)}=\Phi(\vartheta), \Phi^{\circ}(x) \subset B\left(\xi_{x}, \frac{C_{\rho} r_{0}^{|x|}}{1-C_{\rho} r_{0}}\right)$ for all $x \in X$, and $\Phi(x)=\bigcup_{y \in \mathcal{J}_{1}(x)} \Phi(y)$ for all $x \in X$.

Using (VD) and the Lebesgue differentiation theorem, it was proved in Ch that $\mu\left(\bigcup_{x \in \mathcal{J}_{m}} \Phi^{\circ}(x)\right)=\mu(K)$ for all $m$; moreover, for $x \neq y \in X$ with $|x|=|y|$, it follows from $\frac{C_{\rho}^{3} r_{0}}{1-C_{\rho} r_{0}}+C_{\rho}^{2} b \leq \frac{1}{2}$ that $\Phi^{\circ}(x) \cap \Phi^{\circ}(y)=\emptyset$, which implies $\mu(\Phi(x) \cap \Phi(y))=0$.

The original framework of index trees in Definition 3.2, as we will see below, is the natural tree structures on self-similar sets, which are known as the symbolic representations of their associated iterated function systems.

Example 3.4. Self-similar sets Let $\left\{S_{i}\right\}_{i \in \Sigma}$, where $\Sigma$ is a finite set, be an iterated function system (IFS) of contractive similitudes on $\mathbb{R}^{d}$ such that $S_{i}$ has contraction ratio $r_{i} \in(0,1)$. The self-similar set is the unique nonempty compact set $K \subset \mathbb{R}^{d}$ that satisfies $K=\bigcup_{i \in \Sigma} S_{i}(K)$. We assume that the IFS satisfies the open set condition (OSC), i.e., there exists a nonempty bounded open set $O$ such that $S_{i}(O) \subset O$, and $S_{i}(O) \cap S_{j}(O)=\emptyset$ for $i \neq j \in \Sigma$. Let $\rho$ be the Euclidean metric and $\mu$ be the $\alpha$-Hausdorff measure on $K$, where $\alpha$ is the Hausdorff dimension of $K$ (determined by $\sum_{i \in \Sigma} r_{i}^{\alpha}=1$ ).

Let $\Sigma^{*}=\bigcup_{n \geq 0} \Sigma^{n}$ (by convention $\Sigma^{0}=\{\vartheta\}$ ). For $x=i_{1} i_{2} \cdots i_{k} \in \Sigma^{*}$, write $S_{x}:=$ $S_{i_{1}} \circ S_{i_{2}} \circ \cdots \circ S_{i_{k}}$ and $r_{x}:=r_{i_{1}} r_{i_{2}} \cdots r_{i_{k}}$ for short. Let $r_{0}:=\min _{i \in \Sigma} r_{i}$. Define $\mathcal{J}_{0}=\{\vartheta\}$ and for $n \geq 1$,

$$
\mathcal{J}_{m}=\left\{x=i_{1} i_{2} \cdots i_{k} \in \Sigma^{*}: r_{x} \leq r_{0}^{m}<r_{i_{1} i_{2} \cdots i_{k-1}}\right\} .
$$

For $m \geq 1$ and $x \in \mathcal{J}_{m}$, there exists a unique $x^{-} \in \mathcal{J}_{m-1}$ such that $S_{x}(K) \subset S_{x^{-}}(K)$. Naturally we set $X=\bigcup_{m=0}^{\infty} \mathcal{J}_{m}, E_{v}=\left\{\left(x, x^{-}\right),\left(x^{-}, x\right): x \in X, x \neq \vartheta\right\}$ and $\Phi(x)=$ $S_{x}(K)$ for $x \in X$. It is straightforward that $\left(X, E_{v}, \Phi\right)$ is an index tree of $(K, \rho, \mu)$ with contraction ratio $r_{0}$.

Remark. For some self-similar sets we can replace the Hausdorff measure by a selfsimilar measure $\mu$ defined by $\mu(\cdot)=\sum_{i \in \Sigma} p_{i} \mu\left(S_{i}^{-1}(\cdot)\right)$, where $\left\{p_{i}\right\}_{i \in \Sigma}$ is a set of positive probability weights such that $\mu$ is a doubling measure on $K$ (see [Yu]).

An index tree provides a convenient symbolic representation of elements in the underlying space $K$. However, this representation does not reflect the actual geometric structure of $K$. For this reason, we strengthen the index tree by adding a set of horizontal edges in light of the positional relationship among the subsets in each $\Phi\left(\mathcal{J}_{n}\right)$ (see [Ka2, LW1,LW2]). 
Definition 3.5. Let $\left(X, E_{v}, \Phi\right)$ be an index tree of $(K, \rho, \mu)$. For a fixed $\gamma>0$, define a horizontal edge set on $X$ by

$$
E_{h}:=\bigcup_{m=1}^{\infty}\left\{(x, y) \in \mathcal{J}_{m} \times \mathcal{J}_{m}: x \neq y, \operatorname{dist}(\Phi(x), \Phi(y)) \leq \gamma \cdot r_{0}^{m}\right\} .
$$

Let $E=E_{v} \cup E_{h}$. We call $(X, E, \Phi)$ an augmented tree of $(K, \rho, \mu)$.

Remark. The notion of augmented tree was coined by Kaimanovich in Ka2 where the inequality in (3.2) was originally given by $\Phi(x) \cap \Phi(y) \neq \emptyset$. The modified version we define here is adopted from [LW2] in order to avoid the superfluous conditions in [LW1].

Clearly $(X, E)$ is a pre-augmented tree, and the hyperbolicity can be verified using Proposition 2.2 and a similar proof as in [LW2, Theorem 1.2]. On the augmented tree, We extend the Gromov product to the boundary $\partial_{H} X$ by

$$
(x \mid \eta)=\sup \left\{\lim _{n \rightarrow \infty}\left(x \mid y_{n}\right)\right\}, \quad(\xi \mid \eta)=\sup \left\{\lim _{n \rightarrow \infty}\left(x_{n} \mid y_{n}\right)\right\}, \quad x \in X, \xi, \eta \in \partial_{H} X,
$$

where the supremum is taken over all geodesic rays $\pi(\vartheta, \xi)=\left[\vartheta, x_{1}, \cdots\right]$ and $\pi(\vartheta, \eta)=$ $\left[\vartheta, y_{1}, \cdots\right]$; the above limits exist since both $\left(x \mid y_{n}\right)$ and $\left(x_{n} \mid y_{n}\right)$ are increasing in $n$, and the difference of the limits for different rays are at most 1 (see [LW2,, LW] for details). Then $\varrho_{a}(\cdot, \cdot)$ on $\widehat{X}_{H}$ can be defined in the same way as in (2.1), and it is still a quasi-metric.

We define a map $\kappa$ on the collection of geodesic rays starting at $\vartheta$ to $K$ by

$$
\left\{\kappa\left(\left[x_{0}, x_{1}, \cdots\right]\right)\right\}=\bigcap_{n=0}^{\infty} \Phi\left(x_{n}\right) .
$$

For each $\xi \in \partial_{H} X$, it is known that $\kappa(\pi(\vartheta, \xi))$ is independent of the choice of $\pi(\vartheta, \xi)$, and hence $\kappa$ defines a map $\hat{\kappa}$ from $\partial_{H} X$ to $K$ by $\hat{\kappa}(\cdot)=\kappa(\pi(\vartheta, \cdot))$, which has been proved to be a Hölder continuous homeomorphism (see [LW2, Theorem 1.3]). The following is the main reason for introducing the augmented tree.

Theorem 3.6. Let $(X, E, \Phi)$ be an augmented tree of $(K, \rho, \mu)$. Then $(X, E)$ is hyperbolic, and $(K, \rho)$ is Hölder equivalent to the hyperbolic boundary $\left(\partial_{H} X, \varrho_{a}\right)$ in the sense that $\varrho_{a}(\xi, \eta) \asymp \rho(\hat{\kappa}(\xi), \hat{\kappa}(\eta))^{-a / \log r_{0}}$ for all $\xi, \eta \in \partial_{H} X$, where $r_{0}$ is the contraction ratio of $\Phi$.

Remark. This theorem is still valid even if we omit the condition " $\mu$-separation" in Definition 3.2 (see [LW2]).

From this result we can identify $\partial_{H} X$ with $K$. By using the doubling condition of $\mu$ and the triangle inequality of the quasi-metric, we have two elementary results on $\mu(\Phi(x))$ which will play an important role in the study of random walks on the augmented trees.

Proposition 3.7. Let $(X, E, \Phi)$ be an augmented tree of $(K, \rho, \mu)$. Then there is a constant $C>1$ such that

$$
C^{-d(x, y)} \mu(\Phi(x)) \leq \mu(\Phi(y)) \leq C^{d(x, y)} \mu(\Phi(x)), \quad \forall x, y \in X .
$$

Consequently every augmented tree has bounded degree. 
Proposition 3.8. Let $(X, E, \Phi)$ be an augmented tree of $(K, \rho, \mu)$. Define for $\xi \neq \eta \in$ $X \cup K$,

$$
p_{\mu}(\xi, \eta):=\sup \{\mu(\Phi(z)): z \in X \text { and } z \text { lies on some canonical } \pi(\xi, \eta)\} \text {. }
$$

Then the supremum can be reached at a vertex lying on the horizontal segment of some canonical geodesic between $\xi$ and $\eta$, and there exists $C>1$ such that

$$
p_{\mu}(\xi, \eta) \leq C \max \left\{p_{\mu}(\xi, \sigma), p_{\mu}(\sigma, \eta)\right\}
$$

for all different $\xi, \eta, \sigma \in X \cup K$. Moreover, $p_{\mu}(\cdot, \cdot)$ satisfies the estimate

$$
p_{\mu}(\xi, \eta) \asymp V(\xi, \eta):=\mu(B(\xi, \rho(\xi, \eta))), \quad \forall \xi, \eta \in K, \xi \neq \eta .
$$

\section{Reversible random walks on augmented trees}

Let $(X, E, c)$ be a (rooted) network with energy $\mathcal{E}_{X}[f]=\frac{1}{2} \sum_{x, y \in E} c(x, y)|f(x)-f(y)|^{2}, f \in$ $\ell(X)$; it induces a reversible random walk $\left\{Z_{n}\right\}_{n=0}^{\infty}$. For each $x \in X \backslash\{\vartheta\}$, we define the return ratio at $x$ by

$$
\lambda(x):=\frac{\mathbb{P}_{x}\left(\left|Z_{1}\right|=|x|-1\right)}{\mathbb{P}_{x}\left(\left|Z_{1}\right|=|x|+1\right)}=\frac{\sum_{z: x \in \mathcal{J}_{1}(z)} c(x, z)}{\sum_{y: y \in \mathcal{J}_{1}(x)} c(x, y)} .
$$

Theorem 4.1. Let $(X, E, c)$ be a network, and suppose the associated $\left\{Z_{n}\right\}_{n=0}^{\infty}$ satisfies (i) $\inf _{(x, y) \in E} P(x, y)>0$; (ii) $\sup _{x \in X \backslash\{\vartheta\}} \lambda(x)<1$. Then the conductance on $(X, E)$ has strong isoperimetry (SI).

In particular, if the above $\left\{Z_{n}\right\}$ is defined on an augmented tree $(X, E, \Phi)$ and satisfies (i) and (ii), then the hypotheses in Theorem 2.4 are fulfilled. Hence the Martin boundary $\mathcal{M}=\mathcal{M}_{\min }$, and is homeomorphic to the hyperbolic boundary $\partial_{H} X$ as well as the underlying compact set $K$.

The proof of the (SI) is similar to [KLW, Theorem 5.1]. Henceforth, we will identify $K$ with the Martin boundary $\mathcal{M}$. To consider the hitting distribution $\nu$, we introduce a key condition on $\left\{Z_{n}\right\}_{n=0}^{\infty}$ which is satisfied by the $\lambda$-NRW introduced below:

$$
\mathbb{P}_{\vartheta}\left(Z_{\tau_{m}}=x\right)=\mu(\Phi(x)), \quad \forall m \geq 1, x \in \mathcal{J}_{m},
$$

where $\tau_{m}:=\inf \left\{n \geq 0:\left|Z_{n}\right|=m\right\}$ is the first hitting time of $\mathcal{J}_{m}$ by $\left\{Z_{n}\right\}_{n=0}^{\infty}$.

Proposition 4.2. In addition to the assumptions in Theorem 4.1, suppose (HD) is satisfied. Then the hitting distribution $\nu$ equals $\mu$. 
One of our objectives is to obtain some explicit estimates of the Martin kernel and the Naïm kernel. For this we consider a class of reversible random walks with constant return ratio $\lambda$ :

$$
\lambda(x) \equiv \lambda, \quad \forall x \in X \backslash\{\vartheta\},
$$

where $\lambda \in(0,1)$ is a constant. With this hypothesis, a subsequence of $\left\{\left|Z_{n}\right|\right\}_{n=0}^{\infty}$ is seen to be a birth and death chain by counting the time instants only when $\left\{Z_{n}\right\}$ moves upward or downward (see [KLW, Proposition 4.1]). With this in mind we introduce the following key definition.

Definition 4.3. Let $(X, E, \Phi)$ be an augmented tree of $(K, \rho, \mu)$. A reversible random walk $\left\{Z_{n}\right\}$ on $(X, E)$ is said to be $\lambda$-natural $(\lambda$-NRW) if it satisfies

$$
c\left(x, x^{-}\right)=\lambda^{-|x|} \mu(\Phi(x)), \quad \forall x \in X \backslash\{\vartheta\}, \quad \text { and } \quad c(x, y) \asymp c\left(x, x^{-}\right), \quad \forall(x, y) \in E_{h} .
$$

The notion of natural random walks was first introduced and studied in [KLW] for the self-similar sets in Example 3.4. In that case the $\alpha$-Hausdorff measure $\mu$ is the self-similar measure with the "natural weight" $p_{i}=r_{i}^{\alpha}$ where the $r_{i}$ 's are the contraction ratios of the corresponding IFS, and $\mu(\Phi(x))=p_{i_{1}} \cdots p_{i_{n}}$ for $x=i_{1} \cdots i_{n}$. Also in [KLW], we defined a class of quasi-NRW with $\mu$ a self-similar measure, and necessarily $\mu$ is doubling [KLW, Theorem 4.8]. Hence the quasi-NRW is also a NRW in Definition 4.3 ,

It is easy to show that for any $\lambda$-NRW the condition $\left(R_{\lambda}\right)$ is satisfied. Also the bounded degree property of the augmented tree (Proposition 3.7) implies that every augmented tree admits a $\lambda$-NRW, and $\inf _{(x, y) \in E} P(x, y)=\inf _{(x, y) \in E} \frac{c(x, y)}{m(x)}>0$. In view of Theorem 4.1 and Proposition 4.2, we have

Theorem 4.4. Let $(X, E, \Phi)$ be an augmented tree of $(K, \rho, \mu)$ and let $\left\{Z_{n}\right\}_{n=0}^{\infty}$ be a $\lambda-N R W$ on $(X, E)$. Then the Martin boundary, the hyperbolic boundary and $K$ are homeomorphic.

Moreover $\left\{Z_{n}\right\}_{n=0}^{\infty}$ satisfies property (HD), and

$$
F(x, \vartheta)=\lambda^{|x|}, \quad \text { and } \quad F(\vartheta, x) \asymp \mu(\Phi(x)), \quad \forall x \in X,
$$

and the hitting distribution $\nu$ on $K$ is $\mu$.

Theorem 4.5. Let $\left\{Z_{n}\right\}_{n=0}^{\infty}$ be a $\lambda-N R W$ on an augmented tree $(X, E, \Phi)$. Then the ever-visiting probability obeys the estimate

$$
F(x, y) \asymp \lambda^{|x|-(x \mid y)} \mu(\Phi(y)) p_{\mu}(x, y)^{-1}, \quad \forall x, y \in X,
$$

where $p_{\mu}(\cdot, \cdot)$ is defined by (3.6). Consequently the Martin kernel satisfies

$$
K(x, \eta) \asymp \lambda^{|x|-(x \mid \eta)} p_{\mu}(x, \eta)^{-1}, \quad \forall x \in X, \eta \in X \cup K .
$$


For a natural random walk, as $K \approx \mathcal{M}$ and $\mu=\nu$ (Theorem 4.4), we view the Poisson integral $H$ in (2.5) as an operator on all $\mu$-integrable functions on $K$. Let $C(K)$ denote the collection of continuous functions on $K$. Applying the estimate in (4.2), we obtain the same Fatou-type result as in [KL, Corollary 3.2].

Corollary 4.6. Let $\left\{Z_{n}\right\}_{n=0}^{\infty}$ be a $\lambda-N R W$ on an augmented tree $(X, E, \Phi)$. Then for $u \in C(K)$ and $\varepsilon>0$, there exists a positive integer $n_{0}$ such that for any $\xi \in K$,

$$
|H u(x)-u(\xi)| \leq \varepsilon, \quad \forall x \in \bigcup_{m \geq n_{0}} \mathcal{J}_{m} \text { with } \xi \in \Phi(x) .
$$

It is not difficult to obtain $\Theta(x, \eta) \asymp \lambda^{-(x \mid \eta)} p_{\mu}(x, \eta)^{-1}$ for $x \in X, \eta \in K$ from the above and (2.7). However the extension to $x \in X$ to $\xi \in K$ requires more delicate work. By analyzing the limit in (2.8) as in [KLW, Theorem 6.3], we can extend the Naïm kernel estimates to $(K \times K) \backslash \Delta$.

Theorem 4.7. Let $(X, E, \Phi)$ be an augmented tree of $(K, \rho, \mu)$ with contraction ratio $r_{0}$, and let $\left\{Z_{n}\right\}_{n=0}^{\infty}$ be a $\lambda-N R W$ on $(X, E)$. Then the Naïm kernel obeys the estimate

$$
\Theta(\xi, \eta) \asymp \lambda^{-(\xi \mid \eta)} p_{\mu}(\xi, \eta)^{-1}, \quad \forall \xi, \eta \in K, \xi \neq \eta
$$

Consequently, by Theorem 3.6 and Proposition 3.8, we have

$$
\Theta(\xi, \eta) \asymp \frac{1}{V(\xi, \eta) \rho(\xi, \eta)^{\beta}}, \quad \forall \xi, \eta \in K, \xi \neq \eta,
$$

where $V(\xi, \eta):=\mu(B(\xi, \rho(\xi, \eta)))$, and $\beta=\log \lambda / \log r_{0}$.

\section{Induced Dirichlet forms}

We first recall the definition of a Dirichlet form $\mathcal{E}$ on $L^{2}(M, \mu)$, and the regularity of $\mathcal{E}$ where $M$ is equipped with a quasi-metric. The reader can refer to [CF, FOT] for the theory of Dirichlet forms.

Definition 5.1. Let $\mu$ be a positive $\sigma$-finite regular Borel measure on $M$ such that $\operatorname{supp}(\mu)=M$. A Dirichlet form $\mathcal{E}$ with domain $\mathcal{F}$ is a symmetric bilinear form which is non-negative definite, closed, densely defined on $L^{2}(M, \mu)$, and satisfies the Markovian property: $u \in \mathcal{F} \Rightarrow \tilde{u}:=(u \vee 0) \wedge 1 \in \mathcal{F}$ and $\mathcal{E}[\tilde{u}] \leq \mathcal{E}[u]$. (Here $\mathcal{E}[u]:=\mathcal{E}(u, u)$ denote the energy of $u$.)

If further $M$ is given a locally compact separable quasi-metric $\rho$, then we say that a Dirichlet form $\mathcal{E}$ is regular if $\mathcal{F} \cap C_{0}(M)$ is dense in $C_{0}(M)$ with the supremum norm,

and dense in $\mathcal{F}$ with the $\mathcal{E}_{1}^{1 / 2}{ }^{-n o r m}$. It is called local if $\mathcal{E}(u, v)=0$ for $u, v \in \mathcal{F}$ having disjoint compact supports. 
For brevity, we write $L^{2}=L^{2}(K, \mu)$, and use $f_{B}$ to denote $\frac{1}{\mu(B)} \int_{B}$ for any measurable set $B \subset K$ with $\mu(B)>0$. For $s>0$, we define

$$
\mathcal{N}_{2,2}^{s}[u]:=\int_{0}^{\infty} \frac{d r}{r} r^{-2 s} \int_{K} f_{B(\xi, r)}|u(\xi)-u(\eta)|^{2} d \mu(\eta) d \mu(\xi), \quad u \in L^{2}(K, \mu),
$$

and the Besov space $\Lambda_{2,2}^{s}=\Lambda_{2,2}^{s}(K, \rho, \mu)=\left\{u \in L^{2}: \mathcal{N}_{2,2}^{s}(u)<\infty\right\}$ with the associated norm $\|u\|_{\Lambda_{2,2}^{s}}=\|u\|_{L^{2}}+\left(\mathcal{N}_{2,2}^{s}[u]\right)^{1 / 2}$. Clearly $\Lambda_{2,2}^{s+\varepsilon} \subset \Lambda_{2,2}^{s}$ for $\varepsilon>0$, and $\Lambda_{2,2}^{s}$ can be trivial when $s$ is a large value (e.g. for $K=[0,1]^{d} \subset \mathbb{R}^{d}, \Lambda_{2,2}^{1}$ contains only constant functions). As a consequence of Silverstein's Theorem (Theorem 2.5), Theorems 4.1, 4.2 and 4.7, we have

Theorem 5.2. Let $(K, \rho, \mu)$ be a compact space of homogeneous type, and let $\left\{Z_{n}\right\}$ be a $\lambda$-NRW on the augmented tree $(X, E, \Phi)$. Then the induced form $\left(\mathcal{E}_{K}, \mathcal{D}_{K}\right)$ satisfies

$$
\mathcal{E}_{K}[u]:=\mathcal{E}_{X}[H u] \asymp \iint_{K \times K \backslash \Delta} \frac{|u(\xi)-u(\eta)|^{2}}{V(\xi, \eta) \rho(\xi, \eta)^{\beta}} d \mu(\xi) d \mu(\eta) \asymp \mathcal{N}_{2,2}^{\beta / 2}[u], \quad \forall u \in \mathcal{D}_{K},
$$

and $\mathcal{D}_{K}:=\left\{u \in L^{2}: \mathcal{E}_{K}[u]<\infty\right\}=\Lambda_{2,2}^{\beta / 2}$, where $\beta=\log \lambda / \log r_{0}$.

We set $\|u\|_{\mathcal{E}_{K}}=\|u\|_{L^{2}}+\left(\mathcal{E}_{K}[u]\right)^{1 / 2}$. Then $\left(\mathcal{D}_{K},\|\cdot\|_{\mathcal{E}_{K}}\right)$ is a Banach space which is equivalent to $\left(\Lambda_{2,2}^{\beta / 2},\|\cdot\|_{\Lambda_{2,2}^{\beta / 2}}\right)$. By the polarization identity, the energy form defines a symmetric bilinear form. We first consider a situation where $\left(\mathcal{D}_{K},\|\cdot\|_{\mathcal{E}_{K}}\right)$ consists of Hölder continuous functions.

Let $\mathcal{C}^{\delta}$ be the space of Hölder continuous functions on $K$ of order $\delta$ and let $\|u\|_{\mathcal{C}^{\delta}}:=$ $\|u\|_{\infty}+\sup _{\xi, \eta \in K} \frac{|u(\xi)-u(\eta)|}{\rho(\xi, \eta)^{\delta}}$. We define the upper dimension $\bar{d}_{\mu}=\overline{\operatorname{dim}} \mu$ of $\mu$ on $(K, \rho, \mu)$ by

$$
\bar{d}_{\mu}=\inf \left\{\alpha: \exists c>0 \text { s.t. } \mu(B(\xi, r)) \geq c r^{\alpha} \forall \xi \in K \text { and } 0<r \leq 1\right\} .
$$

(Recall that $\operatorname{diam}(K)=1$ by convention.) Using (5.2), it is direct to extend the embedding theorem in GHL to the following.

Proposition 5.3. Let $(K, \rho, \mu)$ be a compact space of homogeneous type. If $\beta>\alpha>\bar{d}_{\mu}$, then

$$
\|u\|_{\mathcal{C}^{(\beta-\alpha) / 2}} \leq C\|u\|_{\Lambda_{2,2}^{\beta / 2}}, \quad \forall u \in L^{2} .
$$

That is, $\Lambda_{2,2}^{\beta / 2} \hookrightarrow \mathcal{C}^{(\beta-\alpha) / 2}$ is an embedding.

Assume the $\lambda$-NRW on the augmented tree of $(K, \rho, \mu)$ has a return ratio $\lambda \in\left(0, r_{0}^{\bar{d}_{\mu}}\right)$, that is, $\beta=\log \lambda / \log r_{0}>\bar{d}_{\mu}$. Then for any $\alpha \in\left(\bar{d}_{\mu}, \beta\right)$ fixed, we have $\mathcal{D}_{K}=\Lambda_{2,2}^{\beta} \subset$ $\mathcal{C}^{(\beta-\alpha) / 2} \subset C(K)$ by Proposition 5.3, and $c\left(x, x^{-}\right) \gtrsim\left(r_{0}^{\alpha} / \lambda\right)^{|x|}$ for all $x \in X$ by (4.3) and (5.2). It follows that

$$
|f(x)-f(y)| \leq C\left(\lambda / r_{0}^{\alpha}\right)^{|x| / 2} \mathcal{E}_{X}[f]^{1 / 2}, \quad \forall f \in \mathcal{D}_{X} \text { and }(x, y) \in E,
$$


and therefore we can define a trace map $\operatorname{Tr}: \mathcal{D}_{X} \rightarrow \mathcal{D}_{K}$ by

$$
(\operatorname{Tr} f)(\xi)=\lim _{n \rightarrow \infty} f\left(x_{n}\right), \quad \xi \in K \text { and } \pi(\vartheta, \xi)=\left[\vartheta, x_{1}, \cdots\right] .
$$

Clearly the above limit is independent of the choice of $\pi(\vartheta, \xi)$ and is uniform on $K$. Next recall that the Poisson integral $H$ in (2.5) maps $\mathcal{D}_{K}$ into $\mathcal{H} \mathcal{D}_{X}$, the class of harmonic functions in $\mathcal{D}_{X}$. We further impose a complete norm $\|\cdot\|_{\mathcal{E}_{X}}$ on $\mathcal{D}_{X}$ by

$$
\|f\|_{\mathcal{E}_{X}}^{2}=\sum_{x \in X}|f(x)|^{2} w^{|x|}+\mathcal{E}_{X}[f]
$$

where $0<w<r_{0}^{\bar{d}_{\mu}}$ is a constant (see [KL, Corollary 3.6]). Applying Corollary 4.6 and (5.3), we obtain the following trace theorem [KL, Section 3].

Theorem 5.4. Let $(K, \rho, \mu)$ be a compact space of homogeneous type, and let $\left\{Z_{n}\right\}_{n=0}^{\infty}$ be a $\lambda$-NRW with ratio $\lambda \in\left(0, r_{0}^{\bar{d}_{\mu}}\right)$ on the augmented tree $(X, E, \Phi)$. Then $\operatorname{Tr}\left(\mathcal{D}_{X}\right)=\mathcal{D}_{K}$, and $\left.\operatorname{Tr}\right|_{\mathcal{H} \mathcal{D}_{X}}=H^{-1}$. Moreover, $\operatorname{Tr}:\left(\mathcal{H D}_{X},\|\cdot\|_{\mathcal{E}_{X}}\right) \rightarrow\left(\mathcal{D}_{K},\|\cdot\|_{\mathcal{E}_{K}}\right)$ is a Banach space isomorphism.

We are interested in conditions under which $\left(\mathcal{E}_{K}, \mathcal{D}_{K}\right)$ is a (non-local) Dirichlet form. It is easy to check the properties in Definition 5.1 except the density property in $L^{2}$ and in $C(K)$. To this end, we introduce two critical exponents of the family $\left\{\Lambda_{2,2}^{\beta / 2}\right\}_{\beta>0}$ by

$$
\begin{aligned}
& \beta^{*}:=\sup \left\{\beta>0: \Lambda_{2,2}^{\beta / 2} \cap C(K) \text { is dense in } C(K) \text { with }\|\cdot\|_{\infty}\right\}, \quad \text { and } \\
& \beta^{\sharp}:=\sup \left\{\beta>0: \operatorname{dim}\left(\Lambda_{2,2}^{\beta / 2} \cap C(K)\right)>1\right\} .
\end{aligned}
$$

As $C(K)$ is dense in $L^{2}(K, \rho, \mu)$, the Proposition 5.4 implies that $\left(\mathcal{E}_{K}, \mathcal{D}_{K}\right)$ is a non-local regular Dirichlet form if $\bar{d}_{\mu}<\beta<\beta^{*}$. In general when $\rho$ is a metric, we know that $2 \leq \beta^{*}\left(\leq \beta^{\sharp} \leq \infty\right)$ (see [St] for $\alpha$-set $K$ ). Indeed, if we let $\mathcal{G}:=\left\{\rho_{\xi}\right\}_{\xi \in K}$ be the class of distance functions on $K$ defined by $\rho_{\xi}(\eta):=\rho(\xi, \eta)$. Then it is straightforward that $\mathcal{G} \subset \Lambda_{2,2}^{\beta / 2} \cap C(K)$ for $\beta \in(0,2)$, and $\mathcal{G}$ separates points in $K$. By the Stone-Weierstrass theorem, $\Lambda_{2,2}^{\beta / 2} \cap C(K)$ is dense in $C(K)$ when $\beta \in(0,2)$, and hence $2 \leq \beta^{*}$. (For $\beta \in(0,2)$, we cannot prove whether $\Lambda_{2,2}^{\beta / 2} \cap C(K)$ is dense in $\Lambda_{2,2}^{\beta / 2}$ with $\|\cdot\|_{\mathcal{E}_{K}}$.) Furthermore, by using the same argument as in GHL, we can show that $\beta^{*}$ has an upper bound, $\beta^{*} \leq \beta^{\sharp} \leq \bar{d}_{\mu}+1$ provided that $(K, \rho)$ satisfies a chain condition in GHL.

The values of $\beta^{*}$ and $\beta^{\sharp}$ are known and are equal for any standard cases: for any classical domain in $\mathbb{R}^{d}$ with Lebesgue measure, $\beta^{*}=\beta^{\sharp}=2$; for the $d$-dimensional Sierpiński gasket with $\alpha$-Hausdorff measure (here $\alpha=\log (d+1) / 2$ is the Hausdorff dimension), $\beta^{*}=\beta^{\sharp}=\log (d+3) / \log 2$ (see [Jo]); for Cantor-type set, $\beta^{*}=\beta^{\sharp}=\infty$. There are also examples that the two exponents $\beta^{*}$ and $\beta^{\sharp}$ are different (see [GuL,, $\left.\mathrm{KL}\right]$ ).

Corresponding to the critical exponents of the domain $\mathcal{D}_{K}$ of the induced energy $\mathcal{E}_{K}$, there are critical values of the return ratio $\lambda$ of the random walk on the augmented tree 
$(X, E)$. Such values can be analyzed, and explicitly calculated in some special cases through the associated electrical network on $(X, E)$. For this, we recall the effective resistances defined as in (2.3); we will fix a set of conductance that defines a $\lambda$-NRW (it can be replaced by any other one in (4.3)) by letting

$$
c\left(x, x^{-}\right)=\lambda^{-|x|} \mu(\Phi(x)), \quad x \in X \backslash\{\vartheta\}, \quad c(x, y)=\frac{c\left(x, x^{-}\right) c\left(y, y^{-}\right)}{c\left(x, x^{-}\right)+c\left(y, y^{-}\right)}, \quad(x, y) \in E_{h} .
$$

Let $\left\{\kappa_{n}\right\}_{n=0}^{\infty}$ be a sequence of mappings from $K$ to $X$ such that for each $\xi \in K$, the sequence $\left[\kappa_{0}(\xi), \cdots, \kappa_{n}(\xi), \cdots\right]$ is a geodesic ray $\pi(\vartheta, \xi)$; we call such $\left\{\kappa_{n}\right\}_{n=0}^{\infty}$ a $\kappa$-sequence. For $n \geq 1$, let $X_{n}:=\bigcup_{k=0}^{n} \mathcal{J}_{k}$, and define the level-n resistance (with respect to $\kappa_{n}$ ) between two nonempty closed subsets $A, B \subset K$ by

$$
R_{n}^{(\lambda)}(A, B):=R_{X_{n}}\left(\kappa_{n}(A), \kappa_{n}(B)\right),
$$

where the conductance on $X_{n} \times X_{n}$ is given by (5.5) . With the assumption $\lambda \in\left(0, r_{0}^{\bar{d}_{\mu}}\right)$, it has been proved that the limit

$$
R^{(\lambda)}(A, B):=\lim _{n \rightarrow \infty} R_{n}^{(\lambda)}(A, B)
$$

exists, and is independent of the choice of the $\kappa$-sequence $\left\{\kappa_{n}\right\}$ [KL, Theorem 4.2]; we call $R^{(\lambda)}(A, B)$ the limit resistance between $A$ and $B$. For $\xi, \eta \in K$, we write $R^{(\lambda)}(\{\xi\},\{\eta\})=$ $R^{(\lambda)}(\xi, \eta)$.

Theorem 5.5. [KL, Theorem 5.1 and Corollary 5.2] Let $(K, \rho, \mu)$ be a compact space of homogeneous type, and let $\left\{Z_{n}\right\}_{n=0}^{\infty}$ be a $\lambda$-NRW with ratio $\lambda \in\left(0, r_{0}^{\bar{d}_{\mu}}\right)$ on the augmented tree $(X, E, \Phi)$. Suppose two nonempty closed subsets $A, B \subset K$ satisfy $R^{(\lambda)}(A, B)>0$. Then

$$
R^{(\lambda)}(A, B)^{-1}=\inf \left\{\mathcal{E}_{K}[u]: u \in \mathcal{D}_{K} \text { with } u=1 \text { on } A, \text { and } u=0 \text { on } B\right\},
$$

and there exists a unique energy minimizer $u_{0}$, i.e., $\left.u_{0}\right|_{A}=1,\left.u_{0}\right|_{B}=0$ and $\mathcal{E}_{K}\left[u_{0}\right]=$ $R^{(\lambda)}(A, B)^{-1}$. In particular, for $\xi, \eta \in K, R^{(\lambda)}(\xi, \eta)>0$ if and only if there exists $u \in \mathcal{D}_{K}$ such that $u(\xi) \neq u(\eta)$; in this case, we have

$$
R^{(\lambda)}(\xi, \eta)=\sup \left\{\frac{|u(\xi)-u(\eta)|^{2}}{\mathcal{E}_{K}[u]}: u \in \mathcal{D}_{K}, \mathcal{E}_{K}[u]>0\right\}
$$

We introduce two critical values for the limit resistances by

$$
\begin{aligned}
& \lambda^{*}:=\inf \left\{\lambda \in\left(0, r_{0}^{\bar{d}_{\mu}}\right): R^{(\lambda)}(\xi, \eta)>0, \forall \xi \neq \eta \in K\right\}, \quad \text { and } \\
& \lambda^{\sharp}:=\sup \left\{\lambda \in\left(0, r_{0}^{\bar{d}_{\mu}}\right): R^{(\lambda)}(\xi, \eta)=0, \forall \xi, \eta \in K\right\} .
\end{aligned}
$$

Then $\lambda^{\sharp} \leq \lambda^{*}$, and the above theorem together with the Stone-Weierstrass theorem yield 
Corollary 5.6. With the same assumption as in Theorem 5.5, if $\lambda^{*}\left(\lambda^{\sharp}\right) \in\left(0, r_{0}^{\bar{d}_{\mu}}\right)$, we have

$$
\beta^{*}=\log \lambda^{*} / \log r_{0}, \quad\left(\beta^{\sharp}=\log \lambda^{\sharp} / \log r_{0}, \text { respectively }\right),
$$

and if $\lambda^{*}=0$, then $\beta^{*}=\infty$.

In general when the cardinality of $K$ is infinity, it is difficult to determine the critical exponents $\beta^{*}$ and $\beta^{\sharp}$ by applying Corollary 5.6 directly, since it is not feasible to test the positivity of limit resistances between every pair of points in $K$. However, when $K$ is a self-similar set, such an infinite exhaustive testing can be reduced to a finite set.

For a self-similar set $K$, we use the same notations as in Example 3.4. Let $i^{\infty} \in K$ denote the unique fixed point of the contractive similitude $S_{i}$ in the IFS $\left\{S_{i}\right\}_{i \in \Sigma}$, i.e., $\left\{i^{\infty}\right\}=\bigcap_{n=1}^{\infty} S_{i^{n}}(K)$.

Theorem 5.7. [KL, Theorem 5.4] Suppose the IFS $\left\{S_{i}\right\}_{i \in \Sigma}$ satisfies the OSC. Let $K$ be the self-similar set equipped with a doubling self-similar measure $\mu$, and let $(X, E, \Phi)$ be the augmented tree defined in Example 3.4 and (3.2). Then

$$
\lambda^{\sharp}=\sup \left\{\lambda \in\left(0, r_{0}^{\bar{d}_{\mu}}\right): R^{(\lambda)}\left(i^{\infty}, j^{\infty}\right)=0, \forall i, j \in \Sigma\right\} .
$$

We say that a self-similar set $K$ is post critically finite (p.c.f.) [Ki1] if it has a finite post critical set $\mathcal{P}$ defined by

$$
\mathcal{P}=\bigcup_{n \geq 1} \sigma^{n}\left(\pi^{-1}\left(\bigcup_{i, j \in \Sigma, i \neq j}\left(S_{i}(K) \cap S_{j}(K)\right)\right)\right),
$$

where $\pi$ is the natural projection from the symbolic space $\Sigma^{\infty}$ to $K$, and $\sigma$ is the shift operator on $\Sigma^{\infty}$. We introduce a geometric condition on the p.c.f. set:

(*) there exist $\delta, c>0$ such that for any $i, j \in \Sigma$ and $\zeta \in S_{i}(K) \cap S_{j}(K)$,

$$
|\xi-\eta| \geq c(|\xi-\zeta|+|\zeta-\eta|), \quad \forall \xi \in S_{i}(K) \cap B(\zeta, \delta) \text { and } \eta \in S_{j}(K) \cap B(\zeta, \delta) .
$$

It is easy to check that the most familiar p.c.f. sets including nested fractals satisfy this condition. Let $V_{0}=\pi(\mathcal{P})$ be the "boundary" of a p.c.f. set $K$.

Theorem 5.8. [KL, Theorem 5.9] With the same assumption as in Theorem 5.7, assume further $K$ is p.c.f. and satisfies (*). Then

$$
\lambda^{*}=\inf \left\{\lambda \in\left(0, r_{0}^{\bar{d}_{\mu}}\right): R^{(\lambda)}(\xi, \eta)>0, \forall \xi \neq \eta \in V_{0}\right\} .
$$

In $[\mathrm{KL}]$ we provided a procedure and examples to implement the above two theorems; the technique is from the network theory including the series and parallel laws, the monotonicity law (cutting and shorting) as well as the $\Delta-Y$ transfrom [DoSn, LyP. We remark that on the same underlying set, there can be different augmented trees which admit 
different random walks and induces different Dirichlet forms. For example for $K=[0,1]$, it is generated by $S_{1}(\xi)=\frac{1}{2} \xi$ and $S_{2}(\xi)=\frac{1}{2}(\xi+1)$ on $\mathbb{R}$. The self-similar measure $\mu$ with doubling property ( $(\mathrm{VD})$ can only be the Lebesgue measure; in this case, $\beta^{*}=\beta^{\sharp}=2$. On the other hand, by using the same method as in $\underline{\mathrm{KL}}$, we see in the following example that for $[0,1]$ generated by another iterated function system, the results can be vastly different.

Example 5.9. Rotated unit interval Let $S_{1}(\xi)=\frac{1}{2} \xi$ and $S_{2}(\xi)=1-\frac{1}{2} \xi$ on $\mathbb{R}$. Then the self-similar set $K$ is the interval $[0,1]$, and is p.c.f. with $\mathcal{P}=\left\{1^{\infty}, 21^{\infty}\right\}$. Let $\mu$ be the self-similar measure generated by probability weights $p, q$. Then $(K, \mu)$ is doubling with respect to the Euclidean metric on $\mathbb{R}$ (see $[\mathrm{Yu}$ ), and its upper dimension is $\bar{d}_{\mu}=-\log (\min \{p, q\}) / \log 2$. The $\lambda^{*}=\lambda^{\sharp}=p q$, and the critical exponents $\beta^{*}=\beta^{\sharp}=-\log (p q) / \log 2$.

To conclude, we remark that the induced Dirichlet forms $\mathcal{E}_{K}^{(\beta)}$ in our study are nonlocal. For local regular Dirchlet forms, which give the Laplacians, are more difficult to obtain. So far in the analysis on fractals, the most studied Laplacians are on the p.c.f. sets and the Sierpiński carpet. It is still an open question for the existence of local Dirichlet form/Laplacian on self-similar sets; in particular, we do not know if there is a non-trivial fractal set that do not support a Laplacian. On the other hand, it is observed that in all the known cases, the local regular Dirichlet forms have Besov spaces $\Lambda_{2, \infty}^{\beta^{*}}$ as domains, and $\Lambda_{2,2}^{\beta^{*}}$ only contain constant functions. The critical exponent conceals lot of information, and it is still far from clear. One of the challenge questions is to understand the "transition" of $\mathcal{E}_{K}^{(\beta)}$ as $\beta \nearrow \beta^{*}$ (see [BBM, GY, Ya]), and in addition, in terms of the random walks in our consideration.

\section{References}

[AM] R. Alvarado, M. Mitrea, Hardy Spaces on Ahlfors-regular quasi metric spaces, a sharp theory, Lecture Notes in Math., No. 2142, Springer, 2015.

[An] A. Ancona, Positive harmonic functions and hyperbolicity, Potential Theory: Surveys and Problems, Lecture Notes in Math., No. 1344, Springer, 1988, 1-23.

[BGPW] A. Bendikov, A. Grigor'yan, C. Pittet, W. Woess, Isotropic Markov semigroups on ultra-metric spaces, Russian Math. Survey 69 (2014), 589-680.

[BBM] J. Bourgain, H. Brezis, P. Mironescu, Another look at Sobolev spaces, Optimal Control and Partial Differential Equations, IOS, Amsterdam, 2001, 439-455.

[CF] Z.Q. Chen, M. Fukushima, Symmetric Markov Processes, Time Changes and Boundary Theory, London Math. Soc. Mono. vol. 35. Princeton University Press, Princeton (2011). 
[Ch] M. Christ, A T (b) theorem with remarks on analytic capacity and the Cauchy integral, Colloq. Math. 60/61 (1990), 601-628.

[CW] R. Coifman, G. Weiss, Extensions of Hardy spaces and their use in analysis, Bull. Amer. Math. Soc. 83 (1977), 569-645.

[DH] D. Deng, Y. Han, Harmonic Analysis on Spaces of Homogeneous Type. Lecture Notes in Math., No. 1966, Springer, 2009.

[DeSa] M. Denker, H. Sato, Sierpiński gasket as a Martin boundary II: The intrinsic metric, Publ. RIMS, Kyoto Univ. 35 (1999), 769-794.

[DoSn] P. Doyle, L. Snell, Random Walks and Electric Networks, The Carus Math. Monogr., vol. 22, 1984.

[Dy] E. Dynkin, Boundary theory of Markov processes (the discrete case), Russian Math. Surveys 24 (1969), 1-42.

[FOT] M. Fukushima, Y. Oshima and M. Takeda, Dirichlet Forms and Symmetric Markov Processes, De Gruyter (2011).

[GHL] A. Grigor'yan, J.X. Hu, K.S. Lau, Heat kernels on metric-measure spaces and an application to semilinear elliptic equations, Trans. Amer. Math. Soc. 355 (2003), 2065-2095.

[GY] A. Grigor'yan, M. Yang, Local and non-local Dirichlet forms on the Sierpiński carpet, arXiv:1706.03318.

[Gr] M. Gromov, Hyperbolic groups, Essays in group theory, Math. Sci. Res. Inst. Publ., vol. 8, Springer, 1987, pp. 75-263.

[GuL] Q.S. Gu, K.S. Lau, Dirichlet forms and critical exponents on fractals, arXiv:1703.07061.

[Jo] A. Jonsson, Brownian motion on fractals and function spaces, Math. Zeit. 222 (1996), 495-504.

[JoWa] A. Jonsson, H. Wallin, Function spaces on subsets of $\mathbb{R}^{n}$, Math. Reports 2, Part 1. Harwood Academic Publ. (1984).

[JuLW] H. Ju, K.S. Lau, X.Y. Wang, Post-critcally finite fractal and Martin boundary, Tran. Amer. Math. Soc. 364 (2012), 103-118.

[Ka1] V. Kaimanovich, Boundaries of invariant Markov operators: the identification problem, Ergodic theory of $Z^{d}$ actions (Warwick, 1993-94), London Math. Soc. Lecture Note Ser. 228, Cambridge Univ. Press, 1996, pp. 127-176.

[Ka2] V. Kaimanovich, Random walks on Sierpiński graphs: hyperbolicity and stochastic homogenization, Fractals in Graz 2001, Trends Math., Birkhuser 2003, pp. $145-183$.

[Ki1] J. Kigami, Analysis on Fractals. Cambridge Tracts in Mathematics vol. 143. Cambridge Univ. Press, 2001. 
[Ki2] J. Kigami, Dirichlet forms and associated heat kernels on the Cantor set induced by random walks on trees, Adv. Math. 225 (2010), 2674-2730.

[KLW] S.L. Kong, K.S. Lau, T.K.L. Wong, Random walks and induced Dirichlet forms on self-similar sets, Adv. Math. 320 (2017), 1099-1134.

[KL] S.L. Kong, K.S. Lau, Critical exponents of induced Dirichlet forms on self-similar sets, submitted, arXiv:1612.01708.

[LN1] K.S. Lau, S.M. Ngai, Martin boundary and exit space on the Sierpinski gasket. Sci. China Math. 55 (2012), 475-494.

[LN2] K.S. Lau, S.M. Ngai, Boundary theory on Hata tree, Nonlinear Analysis 95 (2014), 292-307.

[LW1] K.S. Lau, X.Y. Wang, Self-similar sets as hyperbolic boundaries, Indiana Univ. Math. J. 58 (2009), 1777-1795.

[LW2] K.S. Lau, X.Y. Wang, On hyperbolic graphs induced by iterated function systems, Adv. Math. 313 (2017), 357-378.

[LuS] J. Luukkainen, E. Saksman, Every complete doubling metric space carries a doubling space, Proc. Amer. Math. Soc. 126 (1998), 531-534.

[LyP] R. Lyons, Y. Peres, Probability on Trees and Networks, Cambridge Series in Statistical and Probabilistic Mathematics, vol. 42, Cambridge Univ. Press, 2016.

[MS] R. Macias, C. Segovia, Lipschitz functions on spaces of homogeneous type, Adv. Math. 33 (1979), 257-270.

[Na] L. Naïm, Sur le role de la frontiere de R. S. Martin dans la theorie du potentiel, Ann. Inst. Fourier 7 (1957), 183-281.

[Si] M. Silverstein, Classification of stable symmetric Markov chains, Indiana Univ. Math. J. 24 (1974), 29-77.

[St] A. Stós, Symmetric $\alpha$-stable processes on d-sets, Bull. Polish Acad. Sci. Math. 48 (2000), 237-245.

[Wo] W. Woess, Random Walks on Infinite Graphs and Groups. Cambridge Univ. Press, 2000.

[Ya] M. Yang, Construction of local regular Dirichlet from on the Sierpiński gasket using $\Gamma$-convergence, arXiv:1706.04998v2.

[Yu P.L. Yung, Doubling properties of self-similar measures, Indiana Univ. Math. J. 56 (2007), 965-990.

Shi-Lei Kong, Fakultät für Mathematik, Universität Bielefeld, Postfach 100131, 33501 Bielefeld, Germany. skong@math.uni-bielefeld.de 
Ka-Sing Lau, Department of Mathematics, The Chinese University of Hong Kong, Hong Kong.

\& School of Mathematics and Statistics, Central China Normal University, Wuhan, 430079, China.

\& Department of Mathematics, University of Pittsburgh, Pittsburgh, PA 15260, USA. kslau@math.cuhk.edu.hk

Ting-Kam Leonard Wong, Department of Mathematics, University of Southern California, Los Angeles, CA 90089 USA.

tkleonardwong@gmail.com 